\title{
Composição centesimal, compostos bioativos e atividade antioxidante de frutos de Morinda citrifolia Linn (noni) cultivados no Paraná
}

PALIOTO, G.F.1*; SILVA, C.F.G.'; MENDES, M.P.'; ALMEIDA, V.V.1, ROCHA, C.L.M.S.C.2; TONIN, L.T.D.1 'Universidade Tecnológica Federal do Paraná, campus Apucarana, Rua Marcílio Dias 635, CEP 86812-460, Apucarana-PR; ' Universidade Estadual de Maringá, Avenida Colombo 5.790, CEP: 87020-900, Maringá-PR; *gfppalioto@yahoo.com.br

RESUMO. O fruto de noni (Morinda citrifolia L.) é consumido há milênios na medicina popular polinésia devido aos benefícios nutricionais e terapêuticos. O consumo de noni em outros países, incluindo o Brasil, cresceu vertiginosamente nos últimos anos em decorrência das atividades biológicas atribuídas a ingestão do suco da fruta, principalmente pela propriedade anticâncer. Contudo, a composição química da planta, que está relacionada com suas propriedades biológicas, é determinada pelo seu local de origem, e por influência do clima e do solo onde é cultivada. Neste sentido, este trabalho teve como objetivo analisar a polpa extraída de frutos maduros de noni cultivados em Maringá-PR. A análise da polpa in natura apresentou $89,16 \%$ de umidade, $0,75 \%$ de cinzas, $2,10 \%$ de proteínas, $2,19 \%$ de lipídios e 5,81\% de carboidratos. Dos compostos bioativos, foram analisados antocianinas (1,39 mg.100 g-1 polpa), flavonoides amarelos (13,01 mg.100 g-1 polpa), carotenóides (0,45 mg.100 g $\mathrm{g}^{-1}$ polpa) e vitamina C $(12,16$ $\mathrm{mg} .100 \mathrm{~g}^{-1} \mathrm{polpa}$ ). Para fenólicos totais e atividade antioxidante foram preparados diferentes extratos, sendo que os maiores teores de fenólicos totais foram encontrados no extrato aquoso (1143,56 mg equivalente de ácido gálico (EAG).100 g-1), seguido do extrato etanólico (966,96 mg EAG.100 g-1), metanol/acetona (820,88 mg EAG.100 g-1) e metanólico (306,33 mg EAG.100 g g $^{-1}$. Os melhores resultados para antioxidantes, determinado pelo $\mathrm{EC}_{50}$ - concentração do extrato necessária para reduzir $50 \%$ do radical DPPH, foram encontrados nos extratos metanol/acetona $\left(E_{50}\right.$ de $\left.25,18 \mathrm{mg} \cdot \mathrm{mL}^{-1}\right)$ e metanólico $\left(E_{50}\right.$ de $\left.25,96 \mathrm{mg} \mathrm{mL}^{-1}\right)$. A atividade antioxidante dos frutos pode estar relacionada com o conteúdo de vitamina $C$, uma vez que os extratos com um menor conteúdo de fenóis totais foram aqueles que apresentaram menores valores de $\mathrm{EC}_{50}$.

Palavras-chave: polpa de noni; composição química; compostos fenólicos; atividade antioxidante.

\begin{abstract}
Proximate composition, bioactive compounds and antioxidant activity of fruits of Morinda citrifolia L. (noni) cultivated in Paraná, Brazil. The noni fruit (Morinda citrifolia L.) has been consumed for millennia in the Polynesian folk medicine because of its nutritional and therapeutic benefits. The consumption of the noni fruit in other countries, including Brazil, has increased in recent years because of the biological activities attributed to the ingestion of noni juice, especially the anticancer effect. However, the chemical composition of the plant, which is related to its biological properties, is determined by its geographical origin and is influenced by climate and soil. Therefore, this study aimed to analyze the pulp extracted from noni fruit grown in Maringá, state of Paraná, Brazil. The fresh pulp analysis showed $89.16 \%$ of moisture, $0.75 \%$ of ash, $2.10 \%$ of protein, $2.19 \%$ of fat and $5.81 \%$ of carbohydrates. The bioactive compounds analyzed were anthocyanins (1.39 mg.100 g-1 pulp), yellow flavonoids (13.01 mg.100 g-1 pulp), carotenoids (0.45 mg.100 g-1 pulp) and vitamin C (12.16 mg.100 g ${ }^{-1}$ pulp). For the analysis of total phenols and antioxidant activity, different extracts were prepared. The highest total phenolic contents were found in the aqueous extract (1143.56 mg EAG.100

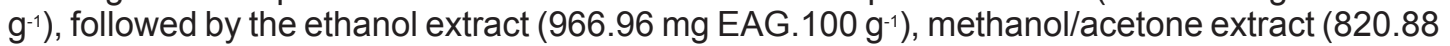
mg EAG.100 $\mathrm{g}^{-1}$ ) and methanol extract (306.33 mg EAG.100 $\left.\mathrm{g}^{-1}\right)$. The results for the antioxidant capacity were determined by the $\mathrm{EC}_{50}$, which is concentration of extract required to reduce $50 \%$ of the DPPH radical. The best results for the antioxidant capacity were found in the methanol/ acetone extract $\left(\mathrm{EC}_{50}\right.$ of $\left.25.18 \mathrm{mg} \cdot \mathrm{mL}^{-1}\right)$ and in the methanol extract $\left(\mathrm{EC}_{50}\right.$ of $\left.25.96 \mathrm{mg} \cdot \mathrm{mL}^{-1}\right)$. The antioxidant activity of the fruit may be related to its vitamin $\mathrm{C}$ content, since the extracts with lower total phenolic content were those that had lower $\mathrm{EC}_{50}$ values.
\end{abstract}

Key words: noni pulp; chemical composition; phenolic compounds, antioxidant activity. 


\section{INTRODUÇÃO}

Produtos naturais e ervas em geral da medicina popular são usadas há séculos em todas as culturas do mundo. Médicos e cientistas se interessam por este campo e reconhecem os benefícios desses compostos à saúde (WANG et al., 2002). O noni (Morinda citrifolia Linn) é uma espécie nativa desde o sudeste da Ásia à Austrália e cultivada na Polinésia, Índia, Caribe, América do Sul e Central. Entre as plantas medicinais utilizadas pelos antepassados na Polinésia o noni vem sendo usado há mais de dois mil anos pela medicina popular da região. Tradicionalmente, a fruta, a folha e a raiz têm sido usadas para prevenir e curar várias doenças devido ao valor terapêutico e nutricional (WANG et al., 2002; SU et al., 2005; CHAN-BLANCO et al., 2006; WEST et al., 2006).

$M$. citrifolia pertence à família Rubiaceae Juss. constituída por, aproximadamente, 6500 gêneros e 13000 espécies (GOVAERTS et al., 2007). Possui distribuição cosmopolita, predominantemente em regiões tropicais, sendo que cerca da metade de suas espécies é de ocorrência neotropical e a maioria encontrada na América do Sul. Nas regiões tropicais ocorre maior representatividade de arbustos e árvores de pequeno porte. No Brasil ocorrem cerca de 130 gêneros com cerca de 1500 espécies (JOLY, 2002). A família é conhecida pela produção de alcaloides, iridoides e antraquinonas (YOUNG et al., 1996), mas são encontrados também taninos, triterpenos e, menos freqüentemente, saponinas (CRONQUIST, 1981; LOPES et al., 2004).

Entre as atividades biológicas de $M$. citrifolia descritas, pode-se citar: atividades antibacteriana, antiviral, anti-helmíntica, analgésica, hipotensora, modulação imunológica, antioxidante, hipoglicemiante, antidepressiva e antiinflamatória (WANG \& SU, 2001; WANG et al., 2002; CHANBLANCO et al., 2006), sendo que a atividade que mais se destaca é a antitumoral (HIRAZUMI et al. 1996, 1994). Como resultado desta reputação, muitos países aumentaram a área para o cultivo de noni, principalmente para a produção do suco do fruto (CHAN-BLANCO et al., 2006).

O noni tem sido objeto de pesquisa sobre suas propriedades nutricionais (KAMIYA et al., 2005; SU et al., 2005; BUI et al., 2006; SAMOYLENKO et al., 2006; DENG et al. 2010; WEST et al. 2011). Aproximadamente 200 compostos fitoquímicos foram identificados, sendo as substâncias mais abundantes os compostos fenólicos, os ácidos orgânicos e os alcalóides. Entretanto, a composição química difere largamente de acordo com a parte da planta (WANG et al., 2002; CHAN-BLANCO et al., 2006).

A composição físico-química completa do fruto ainda não foi determinada, sendo que os trabalhos publicados apresentam diferenças tanto qualitativas quanto quantitativas, como mostra uma comparação feita por Chan-Blanco et al. (2006). Outro fator que determina a composição química da planta é o local de origem, principalmente pela influência do clima e do solo (DENG et al., 2010).

$O$ fruto de noni foi introduzido no Brasil como uma matéria-prima de forte apelo comercial devido a todas as características a ele atribuídas e aos benefícios relacionados ao seu consumo. $O$ cultivo do noni é relatado nos Estados do Acre, São Paulo, Minas Gerais, Pará, Sergipe e Ceará, entre outros (CORREIA et al., 2011). Contudo, poucos são os trabalhos de pesquisa desenvolvidos com essa espécie no país. Como a composição química dos frutos pode variar de acordo com fatores ambientais e distribuição geográfica, torna-se imprescindível estudar a composição do fruto cultivado no Brasil.

Assim, este trabalho teve o objetivo de analisar frutos de noni cultivados na região noroeste do Paraná, com relação às propriedades físico-químicas e compostos bioativos, entre eles compostos fenólicos, carotenóides, antocianinas, flavonoides e vitamina $\mathrm{C}$, assim como a capacidade antioxidante. Buscou-se também avaliar o sistema de solventes, que fornecessem melhor resposta para este último parâmetro.

\section{MATERIAL E MÉTODOS}

\section{Obtenção dos frutos de noni}

Os frutos de noni, maduros, foram coletados em Maringá, Paraná, Brasil, no período de janeiro a julho de 2012. Uma exsicata da espécie está depositada no herbário da Universidade Estadual de Maringá (HUEM), $n^{\circ}$ 25.573. Os frutos foram lavados com água potável corrente e a parte comestível, polpa e casca, separadas manualmente. A polpa preparada foi fragmentada manualmente e congelada em refrigerador doméstico, a $-4{ }^{\circ} \mathrm{C}$, ao abrigo da luz, até o momento das análises.

\section{Preparação dos extratos}

Foram preparados quatro extratos diferentes com a polpa de noni: aquoso $\left(\mathrm{H}_{2} \mathrm{O}\right)$, metanol/água 8:2 (MeOH) e etanol/água 8:2 (EtOH), utilizando-se $100 \mathrm{~mL}$ do solvente extrator com 1,0 g de polpa em agitação magnética ao abrigo da luz. Após 2 horas de agitação, os extratos foram filtrados com papel filtro. O extrato metanol 50\%/acetona $70 \%(\mathrm{MeOH} /$ Ace) foi preparado segundo metodologia descrita por Rufino et al. (2007).

\section{Análises físico-químicas}

As análises físico-químicas foram realizadas

Rev. Bras. PI. Med., Campinas, v.17, n.1, p.59-66, 2015. 
com a polpa fresca. O teor de umidade e cinzas das amostras foi determinado conforme o método descrito pela Association of Official Analytical Chemists (AOAC) (1998), e o teor de proteína bruta segundo o método Kjedahl utilizando o fator de correção igual a 6,25 (INSTITUTO ADOLFO LUTZ, 2005). Os lipídios totais foram extraídos com uma mistura de clorofórmio e metanol (1:2 v/v) (BLIGH \& DYER, 1959). Os carboidratos foram calculados pela diferença: $100-(\%$ umidade $+\%$ cinzas $+\%$ proteína bruta + \% lipídios totais). Todas as análises físico-químicas foram realizadas em triplicatas.

\section{Antocianinas e Flavonoides amarelos}

A determinação de antocianinas totais (ANT) e flavonoides amarelos (FLV) seguiram a metodologia de Francis (1982). Utilizou-se solução extratora de etanol $95 \%+\mathrm{HCl}$ 1,5 mol.L-1 (85:15), procedendo-se a leitura da absorbância a 535 $\mathrm{nm}$ para antocianinas e $374 \mathrm{~nm}$ para flavonoides amarelos. As análises foram realizadas em triplicata, e os resultados foram expressos em mg.100 $\mathrm{g}^{-1} \mathrm{de}$ polpa, calculados através das fórmulas:

ANT = fator de diluição $\times$ absorbância/98,2 FLV = fator de diluição $x$ absorbância/76,6

\section{Carotenóides Totais}

A determinação de carotenoides totais (CRT) foi realizada de acordo com metodologia descrita por Higby (1962), na qual a polpa da noni foi extraída com álcool isopropílico:hexano (3:1). A absorbância foi lida em $450 \mathrm{~nm}$ e o resultado expresso em mg. $100 \mathrm{~g}^{-1}$ de polpa, calculados através da formula:

CRT $=(A \times 100) /(250 \times L \times W)$, onde:

$A=$ absorbância; $L$ = largura da cubeta em $\mathrm{cm} ; \mathrm{W}=$ quociente entre a massa da amostra original em g e o volume final da diluição em $\mathrm{mL}$.

\section{Determinação do teor de ácido ascórbico}

Para a determinação do teor de ácido ascórbico adotou-se a metodologia descrita por Horwitz (2000) e modificada por Benassi \& Antunes (1998). A polpa foi extraída com solução de ácido oxálico $2 \%$ e titulada com 2,6-diclorofenolindofenol $0,01 \%$ até obter-se uma coloração rosa clara. A análise foi realizada em triplicata e os resultados foram expressos como $\mathrm{mg}$ de ácido ascórbico. $100 \mathrm{~g}^{-1}$ da amostra.

\section{Fenólicos totais}

O teor de fenólicos totais dos extratos foi determinado pelo método espectrofotométrico (Lambda 750, Perkin Elmer), com leitura em 725 nm, utilizando-se o reagente Folin-Ciocalteu (SINGLETON \& ROSSI, 1965) e curva padrão de ácido gálico $(y=3,0577 x+0,0445)$. Os resultados foram expressos em mg equivalente de ácido gálico por 100 gramas do extrato (mg EAG.100 $\mathrm{g}^{-1}$ ). Todas as determinações foram realizadas em triplicatas.

\section{Atividade antioxidante}

A atividade antioxidante dos extratos de M. citrifolia foi determinada utilizando-se o método da capacidade de sequestrar o radical 1,1-difenil2-picrilhidrazil (DPPH), descrito por Kulisic et al. (2004). Um mililitro de cada um dos extratos, em diferentes concentrações, foi adicionado a 2,0 mL de uma solução de DPPH $(40 \mu \mathrm{g} / \mathrm{mL} ; 0,10 \mathrm{mM}$ em metanol). Após 30 minutos de incubação, a absorbância foi determinada em espectrofotômetro (Lambda 750, Perkin Elmer) em 515 nm. A atividade antioxidante foi expressa como porcentagem de inibição em relação ao controle negativo, de acordo com a equação:

$\%$ Inibição $=\left[\left(A_{c}-A_{a}\right) / A_{c}\right] \times 100$

$A_{c}=$ absorbância do controle negativo (solução $\mathrm{DPPH}+$ Metanol)

$A_{a}=$ absorbância da amostra após 30 minutos

A concentração do extrato necessária para diminuir a absorbância do DPPH em $50 \%$, $\left(E C_{50}\right)$ foi calculada por regressão linear, utilizandose o programa Microsoft Office Excel 2007. Para cada amostra, uma curva de concentração versus porcentagem de inibição foi obtida. $\mathrm{O}$ antioxidante sintético butil hidroxitolueno (BHT) foi usado como composto de referência. Todas as determinações foram realizadas em triplicatas.

\section{Análise Estatística}

Os resultados apresentados correspondem à média de três repetições \pm desvio padrão. Os resultados foram analisados estatisticamente pelo teste de Tukey $(p<0,05)$, com comparações múltiplas. As análises estatísticas foram realizadas utilizando-se o software GraphPad InStat, Versão 3.02 (1998).

\section{RESULTADOS E DISCUSSÃO}

Os resultados encontrados nas análises de composição centesimal da polpa de noni estão dispostos na Tabela 1.

O resultado do teor de umidade encontrado $(89,16 \%)$ está de acordo com os dados da literatura, que apresenta valores de $90-92 \%$ de água nos frutos de noni (CHAN-BLANCO et al., 2006; CORREIA et al. 2011; WEST et al. 2011). O teor de cinzas, de 0,75\%, mostrou-se dentro da variação encontrada em outros estudos, de 0,4 a 2,1\%, como o de West et al. (2011), de $0,54 \%$, e Correia et al. (2011), de 0,63\%. As cinzas representam os minerais contidos nos alimentos, sendo que os principais elementos encontrados no noni são potássio, enxofre, cálcio e fósforo (CHAN- 
TABELA 1. Composição centesimal da polpa de noni (Morinda citrifolia).

\begin{tabular}{lc}
\hline Análise (\%) & Valores (\%) \\
\hline Umidade & $89,16 \pm 0,04$ \\
Cinzas & $0,75 \pm 0,07$ \\
Proteínas & $2,10 \pm 0,35$ \\
Lipídios & $2,19 \pm 0,44$ \\
Carboidratos & $5,81 \pm 0,77$
\end{tabular}

${ }^{*}$ Resultados expressos como média \pm desvio padrão $(n=3)$.

BLANCO et al., 2006; WEST et al., 2011).

Em relação ao teor de proteínas $(2,10 \%)$ das polpas analisadas, observa-se que foi semelhante ao relatado por Chunhieng (2003), de 2,5\%, em uma análise feita com noni cultivado no Cambodia e de Costa et al. (2013), de 2,24\%, em noni cultivado no Piauí. Este valor médio encontrado foi maior do que os frutos cultivados na Polinésia $(0,55 \%)$ (WEST et al. 2011), Pohnpei $(0,44 \%)$ (SHOVIC \& WHISTLER, 2001) e também dos frutos cultivados no Ceará $(1,06 \%)$ (CORREIA et al., 2011). Segundo os dados de Chan-Blanco et al. (2006), a quantidade de proteína do noni representa $11,3 \%$ da matéria seca, contendo principalmente os aminoácidos glutamina, ácido aspártico e isoleucina.

Os valores de lipídios encontrados em trabalhos com a polpa de noni variam de 0,10 a 0,30\% (SHOVIC \& WHISTLER, 2001; WEST et al., 2011). O nossos resultados estão bem acima desses valores, sendo de $2,19 \%$. Polpas de noni cultivado no Ceará apresentaram 0,08\% de lipídios (CORREIA et al., 2011) e, portanto, valores abaixo daqueles da literatura, sendo que as formas de obtenção, preparo e armazenamento da polpa de noni foram iguais àquelas utilizadas no presente trabalho.

Os carboidratos representaram $5,81 \%$ dos constituintes da polpa de noni, resultado próximo ao apresentado por Correia et al. (2011), que encontrou em valor de $6,32 \%$ na polpa de noni cultivada no Ceará. West et al. (2011) encontrou um valor superior, $7,21 \%$, contendo principalmente glicose, frutose e sucrose.

As análises de antocianinas totais, flavonoides amarelos, carotenóides totais e ácido ascórbico, foram realizadas com a polpa in natura e estão apresentados na Tabela 2.

As antocianinas são pigmentos pertencentes ao grupo dos flavonoides, responsáveis por uma grande variedade de cores de frutas, flores e folhas.
Sua função principal é a proteção da planta contra a luz ultravioleta, evitando assim, a produção de radicais livres (MALACRIDA \& MOTTA, 2006). A polpa de noni apresentou um teor de $1,39 \mathrm{mg} .100$ $\mathrm{g}^{-1}$, um valor muito próximo ao apresentado por Barros et al. (2011), que foi de 1,68 mg.100 g-1. Nos últimos anos, aumentou o interesse pelas antocianinas devido, entre vários outros efeitos fisiológicos, a capacidade antioxidante, propriedade antiinflamatória, prevenção da hiperglicemia e estimulação da secreção da insulina (MILIAUSKAS et al., 2004)

O teor de carotenóides encontrado para a polpa de noni estudada foi de $0,45 \mathrm{mg} 100 \mathrm{~g}^{-1}$, valor muito inferior ao relatado por Costa et al. (2013), que obteve $3,90 \mathrm{mg} 100 \mathrm{~g}^{-1}$ de polpa. Este resultado foi superior ao encontrado por Lima et al. (2007) para a amêndoa do pequi (Caryocar brasiliense), de 0,295 $\mathrm{mg} .100 \mathrm{~g}^{-1}$. As formas mais comuns de carotenóides encontradas nos alimentos são a e $\beta$-caroteno, além do licopeno e luteína. Os carotenóides podem ser encontrados em vegetais folhosos ou não, como taioba e cenoura, frutas, como acerola e cajá, e vegetais frutos como abóbora e tomate (RODRIGUEZ-AMAYA et al., 2008).

Os carotenóides, juntamente com as vitaminas, são os compostos mais estudados como agentes quimo-preventivos, pois atuam como agentes antioxidantes (POOL-ZOBEL et al., 1997). Estudos mostram a relação entre o aumento no consumo de alimentos ricos em carotenóides e a diminuição no risco de várias doenças. Segundo Olson (1999), os carotenóides seqüestram o oxigênio singlete, removem os radicais peróxido, modulam o metabolismo carcinogênico, inibem a proliferação celular, estimulam a comunicação entre células (junções gap) e aumentam a resposta imune.

Com relação aos flavonoides, eles constituem uma importante classe dos polifenóis, presentes em relativa abundância entre os metabólitos secundários das plantas. Esses compostos possuem diversas atividades biológicas, entre elas, propriedade anticarcinogênica atribuída a alguns compostos (KATSUBE et al., 2004; SIMÕES et al., 1999). O conteúdo de flavonoides amarelos encontrado para a polpa de noni foi de 13,01 mg.100 $\mathrm{g}^{-1}$, valor muito superior ao encontrado por Silva et al. (2012) para polpa de diferentes genótipos de cajá (1,37-5,25 mg.100 g-1). Não foram encontrados na literatura dados de flavonóides para frutos de noni

TABELA 2. Valores de antocianinas totais (ANT), flavonoides amarelos (FLV), carotenóides totais (CRT) e ácido ascórbico (Vit. C), para a polpa de noni (Morinda citrifolia).

\begin{tabular}{c|c|c|c}
\hline ANT $^{*}$ & FLV $^{*}$ & CRT $^{*}$ & Vit. $^{*}$ \\
\hline $1,39 \pm 0,06$ & $13,01 \pm 0,40$ & $0,45 \pm 0,01$ & $12,16 \pm 0,51$ \\
\hline
\end{tabular}

${ }^{*} \mathrm{mg} .100 \mathrm{~g}^{-1}$ polpa

Rev. Bras. PI. Med., Campinas, v.17, n.1, p.59-66, 2015. 
cultivados em outras regiões.

O teor de vitamina C, de 12,16 mg. 100 $\mathrm{g}^{-1}$ encontrado nesta análise foi inferior aos apresentados por Correia et al. (2011), de 122,54 mg.100 g ${ }^{-1}$, Shovic \& Whistler (2001), de 155 mg.100 $\mathrm{g}^{-1}$ e West et al. (2011), que obtiveram $113 \mathrm{mg}^{100} \mathrm{~g}^{-1}$. Este resultado ficou próximo de Costa et al. (2013) que encontrou um teor de vitamina $\mathrm{C}$ de 23,1 mg.100 $\mathrm{g}^{-1}$, em frutos cultivados no Piauí. Iloki Assanga et al. (2013) encontraram níveis diferentes de vitamina C de acordo com a maturação e o período de colheita do fruto de noni. $\mathrm{O}$ conteúdo de vitamina $\mathrm{C}$ no noni decai com a maturação do fruto, como demonstrado pelo estudo de Silva et al. (2009) que encontrou valores de 101 a $38 \mathrm{mg} .100 \mathrm{~g}^{-1}$, sendo que os menores valores foram obtidos nos frutos maduros. Chan-Blanco et al. (2007) também encontraram um decaimento na concentração de vitamina $C$, sendo que o fruto imaturo apresentou o maior valor, de 391 mg. $100 \mathrm{~g}^{-1}$, e o fruto com seis semanas de maturação apresentou teor de $148 \mathrm{mg} .100 \mathrm{~g}^{-1}$. Outras frutas e vegetais também apresentam variações de vitamina C durante a maturação, como cerejas (SERRANO et al., 2005), blueberry (CASTREJON et al., 2008) e pimenta vermelha (ZHANG e HAMMAUZU, 2003).

A Tabela 3 apresenta os resultados obtidos para atividade antioxidante e compostos fenólicos dos quatro extratos preparados: aquoso, metanólico $(\mathrm{MeOH})$, etanólico $(\mathrm{EtOH})$ e metanol-acetona (MeOH/Ace).

Comparando a eficiência do solvente de extração, a partir da Tabela 3, pode-se constatar que o extrato aquoso apresentou maior valor para compostos fenólicos da polpa de noni. Segundo Chirinos et al. (2007), a água como solvente extrator pode resultar em extratos com alta impureza (ácidos orgânicos, açúcares, proteínas solúveis) e pode interferir na quantificação destes compostos. Wu et al. (2004) e Vieira et al. (2011) avaliaram o conteúdo de polifenóis de frutas consumidas nos Estados Unidos e no Brasil, respectivamente, e observaram que a fração hidrofílica possuía uma quantidade bem superior desses constituintes que a porção lipofílica. Barros et al. (2011) também observaram um maior conteúdo de fenóis para o extrato aquoso (84 mg EAG.100 $\mathrm{g}^{-1}$ ) quando comparado com o extrato alcoólico (58 mg EAG.100 $\mathrm{g}^{-1}$ ) para a polpa da noni, porém seus resultados foram inferiores aos relatados neste trabalho.

Os resultados de fenóis totais obtidos foram superiores àqueles apresentados por ChanBlanco et al. (2007) e por Correia et al. (2011), que obtiveram valores de 51,1 mg EAG.100 g-1 e 216, 67 mg EAG.100 $\mathrm{g}^{-1}$, respectivamente, para a polpa in natura de noni. Krishnaiah et al. (2013) trabalharam com a polpa de noni desidratada e obtiveram um teor de compostos fenólicos de 431,8 mg EAG.100 g-1. Em outro estudo, Costa et al. (2013) encontraram um teor de compostos fenólicos para os extratos acetônico de 109,81 mg EAG.100 g-1, etanólico de $20,33 \mathrm{mg}^{\mathrm{E} A G} .100 \mathrm{~g}^{-1}$ e aquoso de $12,75 \mathrm{mg}$ EAG.100 $\mathrm{g}^{-1}$.

Vasco et al. (2008) e Rufino et al. (2010), que estudaram o conteúdo de fenólicos totais em diversos frutos, classificaram os frutos em três categorias: baixo (<100 mg EAG/g), médio (100-500 $\mathrm{mg} E A G / g)$ e alto $\mathrm{mg} \mathrm{EAG/g} \mathrm{(>500} \mathrm{mg} \mathrm{EAG/g).} \mathrm{De}$ acordo com essa classificação, os extratos aquoso, $\mathrm{EtOH}$ e $\mathrm{MeOH} /$ Ace são considerados com alto teor de compostos fenólicos. O extrato $\mathrm{MeOH}$ é considerado de médio teor.

As discrepâncias nos valores de teores dos compostos fenólicos, segundo Soares (2008), podem ser influenciadas por diversos fatores, tais quais maturação, espécie, práticas de cultivo, origem geográfica, estágio de crescimento, condições de colheita e processo de armazenamento das frutas. Essas informações estão de acordo com os resultados encontrados por lloki Assanga et al. (2013), onde o conteúdo fenólico dos frutos de noni variou com os estágios de maturação e as estações climáticas, sendo que os maiores teores foram encontrados nos frutos maduros coletados na primavera/verão e no outono. Isto vem a corroborar com os resultados deste trabalho, pois os frutos de noni foram coletados durante o verão e o outono e

TABELA 3. Resultados dos compostos fenólicos totais e da atividade antioxidante $\left(\mathrm{EC}_{50}\right)$ de diferentes extratos de polpa de noni (Morinda citrifolia).

\begin{tabular}{lll}
\hline Extratos & Fenólicos totais $\left(\mathbf{m g}\right.$ EAG.100 $\left.\mathbf{~ g}^{-1}\right)$ & $\mathbf{E C}_{50}\left(\mathbf{m g} \cdot \mathbf{m L}^{-1}\right)$ \\
\hline Aquoso & $1143,56 \pm 36,02^{\mathrm{a}}$ & $45,15 \pm 1,87^{\mathrm{a}}$ \\
$\mathrm{MeOH}$ & $306,33 \pm 22,97^{\mathrm{d}}$ & $25,96 \pm 0,52^{\mathrm{b}}$ \\
$\mathrm{EtOH}$ & $966,96 \pm 42,05^{\mathrm{b}}$ & $45,63 \pm 1,64^{\mathrm{a}}$ \\
$\mathrm{MeOH} /$ Ace & $820,88 \pm 17,31^{\mathrm{c}}$ & $25,18 \pm 1,90^{\mathrm{b}}$ \\
$\mathrm{BHT}$ & - & $0,02 \pm 0,0007$ \\
\hline
\end{tabular}

Resultados expressos como média \pm desvio padrão $(n=3)$. Letras iguais na mesma coluna indicam que não há diferenças significativas $(p<0,05)$ pelo teste de Tukey.

Rev. Bras. PI. Med., Campinas, v.17, n.1, p.59-66, 2015. 
apresentaram elevados níveis de conteúdo fenólico. Os compostos fenólicos estão relacionados com o sabor, coloração, vida de prateleira e ação do produto como um alimento funcional, fortemente correlacionado com a capacidade antioxidante (CORREIA et al., 2011). Segundo Chan-Blanco et al. (2006), os compostos bioativos mais abundantes no noni são os fenólicos, como o damanacantal, escopoletina, morindona e rubiadina, sendo que o damanacantal possui propriedade anti-carcinogênica comprovada (THANI et al., 2010). Chan-Blanco et al. (2007) encontraram no extrato aquoso da polpa de noni os compostos fenólicos rutina e a escopoletina como componentes majoritários.

Muitos compostos fenólicos possuem atividade antioxidante, anticarcinogênica, antimutagênica e anti-inflamatória. Contudo, o principal interesse nos compostos fenólicos está relacionado com sua atividade antioxidante, sendo que as funções fisiológicas e farmacológicas atribuída aos compostos fenólicos podem ter origem nesta propriedade. Muitas plantas que possuem propriedade anti-câncer contêm uma variedade de compostos fenólicos, com diferentes características estruturais, e possuindo diferentes formas de atividade antioxidante (CAI et al., 2006).

O método para determinação da atividade antioxidante, utilizado neste trabalho, consiste na redução do radical DPPH (púrpura) que, ao receber um elétron ou um radical hidrogênio, muda sua coloração para amarelo, ficando estável. O desaparecimento da coloração pode ser avaliado pelo decréscimo da absorbância. Quanto menor o valor de $\mathrm{EC}_{50}$ (concentração de extrato em mg.mL-1 capaz de reagir com $50 \%$ do radical presente na solução de DPPH) maior é sua atividade antioxidante, pois será necessária uma menor quantidade de extrato para reduzir $50 \%$ do radical livre DPPH.

Os resultados apresentados na Tabela 3 mostram que os extratos metanólico e metanolacetona apresentaram maior atividade antioxidante, não diferindo significativamente $(p<0,05)$. O extrato aquoso e etanólico não foram tão eficientes e também não diferiram significativamente $(p<0,05)$. Os resultados demonstram a importância de testarmos diferentes solventes na extração de fitoquímicos, já que, para avaliar a atividade antioxidante de um vegetal, é necessário extrair o máximo de compostos bioativos, e estes podem apresentar polaridade diferenciada. Dessa forma, a solubilidade em um determinado solvente é característica peculiar de cada fitoquímico, o que explica a inexistência de um procedimento de extração universal (MELO et al., 2008).

Os resultados de atividade antioxidante obtidos pelo método DPPH não caracterizam o noni como um bom agente antioxidante quando comparados a outros estudos que utilizaram o mesmo método de análise. Costa et al. (2013) obtiveram valores de $\mathrm{EC}_{50}$ de $1,40 \mu \mathrm{g} \cdot \mathrm{mL}^{-1}$ para o extrato aquoso, $40,98 \mu \mathrm{g} \cdot \mathrm{mL}^{-1}$ para o extrato etanólico e 507,50 $\mu \mathrm{g} \cdot \mathrm{mL}^{-1}$ para o extrato acetônico da polpa de noni cultivada no Piauí. Vieira et al. (2011) estudaram a atividade antioxidante das polpas das frutas tropicais acerola, caju, goiaba, cajá, tamarindo e bacuri. A capacidade antioxidante destas polpas $\left(E_{50}\right.$ em $\left.\mu \mathrm{g} \cdot \mathrm{mL}^{-1}\right)$ variou de 24,42 a 4700,24 para o extrato aquoso e de 1,74 a 2356,96 para o extrato hidroalcoólico. Roesler et al. (2007), avaliaram a capacidade antioxidante de frutas do cerrado brasileiro, e encontraram valores de $\mathrm{EC}_{50}$ (em $\mu \mathrm{g} \cdot \mathrm{mL}^{-1}$ ), para os extratos aquosos e etanólicos da polpa, respectivamente, de 879,93 e 387,47 para cagaita, 1.321,93 e 148,82 para o araticum, 199,34 e 162,97 para lobeira e 534,43 e 298,75 para pequi.

A atividade antioxidante dos extratos não está relacionada com o conteúdo de fenóis totais, já que aqueles que apresentaram maior concentração de fenólicos (aquoso e etanólico) foram àqueles que apresentaram menor atividade antioxidante. A baixa atividade antioxidante da polpa de noni em estudo pode ser atribuída ao baixo teor de vitamina $C$ presente, já que a vitamina $\mathrm{C}$ proporciona proteção contra a oxidação descontrolada no meio aquoso da célula devido ao seu alto poder redutor (COUTO \& CANNIATTI-BRAZACA, 2010), bem como ao baixo teor de carotenóides, que também possuem ação antioxidante (POOL-ZOBEL et al., 1997).

Considerando os dados obtidos e a discussão com os dados da literatura, as diferenças observadas nos frutos de noni analisados podem ser decorrentes do impacto dos fatores ambientais locais, como solo, radiação, temperatura, umidade, corrente de ar, e também das características do fruto, como ponto de maturação e condições de armazenamento (CHAN-BLANCO et al., 2006; DENG et al., 2010; ILOKI ASSANGA et al., 2013).

\section{AGRADECIMENTO}

Os autores são gratos pelo financiamento oriundo da Fundação Araucária do Paraná, convênio $387 / 2012$, e pelas contribuições ao manuscrito de Adriana Lenita Meyer Albiero e Rúbia Michele Suzuki.

\section{REFERÊNCIAS}

ASSOCIATION OF OFFICIAL ANALYTICAL CHEMISTS (AOAC). Official methods of analysis of the association of official analytical chemists. $16^{\text {th }}$ ed. Arlington: AOAC, 1998.

Rev. Bras. PI. Med., Campinas, v.17, n.1, p.59-66, 2015. 
BARROS, N.V.A. et al. Determinação de compostos bioativos na polpa e farinha do noni (Morinda citrifolia Linn.). Nutrire, v.36, p.260-260, 2011.

BENASSI, M.D.; ANTUNES, A.J. A comparison of metaphosphoric and oxalic acids as extractants solutions for the determination of vitamin $C$ in selected vegetables. Arquivos de Biologia e Tecnologia, v.31, n.4, p.503-507, 1998.

BLIGH, F.G., DYER, W.J. A rapid method of total lipid extraction and purification. Canadian Journal of Biochemistry and Physiology, v.37, p.911-917, 1959.

BUI, A.K.T.; BACIC, A.; PETTOLINO, F. Polysaccharide composition of the fruit juice of Morinda citrifolia (Noni). Phytochemistry, v.67, p.1271-1275, 2006.

CAI, Y.Z. et al. Structure-radical scavenging activity relationships of phenolic compounds from traditional Chinese medicinal plants. Life Sciences, v.78, p.28722888, 2006.

CASTREJON, A.D.R. et al. Phenolic profile and antioxidant activity of highbush blueberry (Vacciniumcorymbosum L.) during fruit maturation and ripening. Food Chemistry, v.109, p.564-572, 2008.

CHAN-BLANCO, Y. et al. The noni fruit (Morinda citrifolia L.): A review of agricultural research, nutritional and therapeutic properties. Journal of Food Composition and Analysis, v.19, p.645-654, 2006.

CHAN-BLANCO, Y. et al. The ripening and aging of noni fruits (Morinda citrifolia L.): microbiological flora and antioxidant compounds. Journal of the Science of Food and Agriculture, v.87, n.9, p.1710-1716, 2007.

CHIRINOS, R. et al. Optimization of extraction conditions of antioxidant phenolic compounds from mashua (Tropaeolum tuberosum Ruiz \& Pavon) Tubers. Separation and Purification Technology, v.55, p.217225, 2007.

CHUNHIENG, M.T. Développement de nouveaux aliments sante tropicale: application à la noix du Brésil Bertholettia excelsa et au fruit de Cambodge Morinda citrifolia. 2003, 181p. Tese (Doutorado) - Institut National Polytechnique de Lorraine, France.

CORREIA, A.A.S. et al. Caracterização química e físicoquímica da polpa do noni (Morinda citrifolia) cultivado no estado do Ceará. Alimentos e Nutrição, v.22, n.4, p.609-615, 2011.

COSTA, A.B. et al. Atividade antioxidante da polpa, casca e sementes do noni (Morinda citrifolia Linn). Revista Brasileira de Fruticultura, v.35, n.2, p.345-354, 2013.

COUTO, M.A.L.; CANNIATTI-BRAZACA, M.A.L. Quantificação de vitamina $C$ e capacidade antioxidante de variedades cítricas. Ciência e Tecnologia de Alimentos, v.30, p.15-19, 2010.

CRONQUIST, A. An integrated system of flowering plants. New York: Columbia University Press, 1981. $1262 p$.

DENG, S.; WEST, B.J.; JENSEN, J.C. A quantitative comparison of phytochemical components in global noni fruits and their commercial products. Food Chemistry, v.122, p.267-270, 2010.

FERGUSON, L.R.; PHILPOTT, M.; KARUNASINGHE, $\mathrm{N}$. Dietary cancer and prevention using antimutagens. Toxicology, v.198, p.147-159, 2004.

FRANCIS, F.J. Analysis of anthocyanins. In: MARKAKIS, P. (ed.) Anthocyanins as food colors. New York:
Academic Press, 1982. p.181-207.

GOVAERTS, R. et al. World checklist \& bibliography of Rubiaceae. The Trustees of the Royal Botanic Gardens: Kew, 2007.

GRAPHPAD INSTAT. Software. Versão 3.02. 1998.

HIGBY, W.K. A simplified method for determination of some the carotenoid distribution in natura and carotene - fortified orange juice. Journal of Food Science, v.27, p.42-49, 1962.

HIRAZUMI, A. et al. Anti cancer activity of Morinda citrifolia on intraperitoneally implanted Lewis lung carcinoma in syngenic mice. Proceedings of the Western Pharmacological Society, v.37, p.145-146, 1994.

HIRAZUMI, A. et al. Immunomodulation contributes to the anticancer activity of Morinda citrifolia (Noni) fruit juice. Proceedings of the Western Pharmacological Society, v.39, p.7-9, 1996.

HORWITZ, W. (Ed.) Official Methods of Analysis of the Association of Official Analytical Chemists. $17^{\text {th }}$ ed. $2^{\circ}$ vol. Gaithersburg: AOAC, 2000.

ILOKI ASSANGA, S.B et al. Effect of maturity and harvest season on antioxidant activity, phenolic compounds and ascorbic acid of Morinda citrifolia L. (noni) grown in Mexico (with track change). African Journal of Biotechnology, v.12, p.4630-4639, 2013.

INSTITUTO ADOLFO LUTZ. Métodos químicos e físicos para análise de alimentos. $5^{\mathrm{a}}$ ed. São Paulo, 2005.

JOLY, A.B. Botânica: introdução à taxonomia vegetal. São Paulo: Companhia Editora Nacional, 2002. 777p.

KAMIYA, K. et al. New anthraquinone and iridoid from the fruits of Morinda citrifolia. Chemical and Pharmaceutical Bulletin, v.53, n.12, p.1597-1599, 2005.

KATSUBE, N. et. al. Induction of apoptosis in cancer cells by bilberry (Vaccinium mirtillus) and the anthocyanins. Journal Agriculture Food Chemistry, v.51, p.68-75, 2003.

KITTS, D.D. Bioactive substances in food: identification and potential uses. Canadian Journal of Physiology and Pharmacology, v.72, p.423-424, 1994.

KRISHNAIAH, D. et al. Antioxidant activity and total phenolic content of an isolated Morinda citrifolia L. methanolic extract from Poly-ethersulphone (PES) membrane separator. Journal of King Saud University - Engineering Sciences, in press, 2013.

KULISIC, T. et al. Use of different methods for testing antioxidative activity of oregano essential oil. Food Chemistry, v.85, p.633-640, 2004.

LIU, R.H. Whole grain phytochemicals and health. Journal of Cereal Science, v.46, p.207-219, 2007.

LOPES, S.; et al. Taxonomic significance of alkaloids and iridoid glucosides in the tribe Psychotrieae (Rubiaceae). Biochemical Systematics and Ecology, v.32, p.11871195, 2004.

MALACRIDA, C. R.; MOTTA, S. Antocianinas em suco de uva: composição e estabilidade. Boletim do Centro de Pesquisa de Processamento de Alimentos, v.24, n.1, p.59-82, 2006.

MELO, E.A. et al. Capacidade antioxidante de frutas. Brazilian Journal of Pharmaceutical Sciences, v.44, p.193-201, 2008.

MILIAUSKAS, G.; VENSKUTONIS, P. R.; VAN BEEK, T. A. Secreening of radical scavening activity of some

Rev. Bras. PI. Med., Campinas, v.17, n.1, p.59-66, 2015. 
medicinal and aromatic plant extracts. Food Chemistry, v.85, p.231-237, 2004.

OLSON, J. A. Carotenoids and human health. Archivos Latinoamericanos de Nutrición, v.49, p.7-11, 1999.

POOL-ZOBEL, B.L. et al. Consumption of vegetables reduces genetic damage in humans: fi rst results of a human intervention trial with carotenoid-rich foods. Carcinogenesis, v.18, n.9, p.1847-1850, 1997.

RODRIGUEZ-AMAYA, D.B.; KIMURA, M.; AMAYAFARFAN, J. Fontes de carotenóides: tabela brasileira de composição de carotenóides em alimentos. Brasília: Ministério de Meio Ambiente/Secretaria de Biodiversidade e Florestas, 2008. 99p.

ROESLER, R. et al. Atividade antioxidante de frutas do cerrado. Ciência e Tecnologia de Alimentos, v.27, p.53-60, 2007.

RUFINO, M.S.M. et al. Metodologia cientifica: determinacao da atividade antioxidante total em frutas pela captura do radical livre DPPH. Comunicado Técnico on line - Embrapa, p.1-4, 2007.

RUFINO, M.S.M. et al. Bioactive compounds and antioxidant capacities of 18 non-traditional tropical fruits from Brazil. Food Chemistry, v.121, p.996-1002, 2010.

SAMOYLENKO, V. et al. New constituinte from noni (Morinda citrifolia) fruit juice. Journal of Agricultural and Food Chemistry, v.54, p.6398-6402, 2006.

SERRANO, M. et al. Chemical constituents and antioxidant activity of sweet cherry at different ripening stage. Journal of Agricultural and Food Chemistry, v.53, p.2741-2745, 2005.

SHOVIC, A.C.; WHISTLER, W.A. Food sources of provitamin $A$ and vitamin $C$ in the American Pacific. Tropical Science, v.41, p.199-202, 2001.

SILVA, F.V.G. et al. Bioactive compounds and antioxidant activity in fruits of clone and ungrafted genotypes of yellow mombin tree. Ciência e Tecnologia de Alimentos, v.32, p.685-691, 2012.

SILVA, L.R.D. et al. Caracterização físico-química do fruto de Noni (Morinda citrifolia L.). Horticultura brasileira, v.27, p.S267-S271, 2009.

SIMÕES, C.M.O. et al. Farmacognosia: da planta ao medicamento. Porto Alegra/Florianópolis: Ed. Universidade/UFRGS e Ed. Da UFSC, 1999. 821p.

SINGLETON, V.L.; ROSSI, J.A.J. Colorimetry of total phenolics with phophomolybdic-phosphotungstic acid reagents. American Journal of Enology and Viticulture, v.16, p.144-158, 1965.

SOARES, M. et al. Compostos fenólicos e atividade antioxidante da casca de uvas niágara e isabel. Revista Brasileira de Fruticultura, v.30, p.59-64, 2008.

SU, B.N. et al. Chemical constituents of the fruits of Morinda citrifolia (noni) and their antioxidant activity. Journal of Natural Products, v.68, n.4, p.592-595, 2005.

THANI, W. et al. Anti-proliferative and antioxidative activities of thai noni/yor (Morinda citrifolia linn.) leaf extract. The Southeast Asian Journal of Tropical Medicine and Public Health, v.41, n.2, p.482-489, 2010

VASCO, C.; RUALES, J.; KAMAL-ELDIN, A. Total phenolic compounds and antioxidant capacities of major fruits from Ecuador. Food Chemistry, v.111, p.816-823, 2008.

VIEIRA, L.M. et al. Fenólicos totais e capacidade antioxidante in vitro de polpas de frutos tropicais. Revista Brasileira de Fruticultura, v.33, p.888-897, 2011.

YOUNG, M.C.M. et al. Chemosystematic markers of Rubiaceae. Opera Botanica Belgica, v.7, p.205-212, 1996.

WANG, M.Y.; SU, C. Cancer preventive effect of Morinda citrifolia (Noni). Annals New York Academy of Sciences, v.952, p.161-168, 2001.

WANG, M.Y. et al. Morinda citrifolia (Noni): a literature review and recent advances in Noni research. Acta Pharmacologica Sinica, v.23, n.12, p.1127-1141, 2002.

WEST, B.J. et al. A safety review of noni fruit juice. Journal of Food Science, v.71, n.8, p.100-106, 2006.

WEST, B.J.; DENG, S.; C. JENSEN C.J. Nutrient and phytochemical analyses of processed noni puree. Food Research International, v.44, p.2295-2301, 2011.

WU, X. et al. Lipophilic and hydrophilic antioxidant capacities of common foods in the United States. Journal of Agricultural and Food Chemistry, v.52, p.4026-4037, 2004.

ZHANG, D.; HAMMAUZU, Y. Phenolic compounds, ascorbic acid, carotenoids and a antioxidant properties of green, red and yellow bell peppers. Journal of Food Agriculture and Environment, v.1, n.2, p.22-27, 2003.

Rev. Bras. PI. Med., Campinas, v.17, n.1, p.59-66, 2015. 